\title{
Aurora A Kinase/Tyrosine Kinase Inhibitor ENMD-2076
}

National Cancer Institute

\section{Source}

National Cancer Institute. Aurora A Kinase/Tyrosine Kinase Inhibitor ENMD-2076. NCI

Thesaurus. Code C78190.

An orally bioavailable synthetic small molecule with potential antiangiogenic and antineoplastic activities. Aurora A kinase/tyrosine kinase inhibitor ENMD-2076 selectively binds to and inhibits non-specified tyrosine kinases and Aurora kinases (AKs). The inhibition of AKs may result in the inhibition of cell division and proliferation and may induce apoptosis in tumor cells that overexpress AKs; antiang iogenic activity is related to the inhibition of angiogenic tyrosine kinases. AKs are serine-threonine kinases that play an essential role in mitotic checkpoint control during mitosis and are important regulators of cell division and proliferation. 